\title{
Interfacial Crack Problem of a Class of Spliced Materials
}

\author{
Minhai Huang \\ College of Mathematics and Statistics, Zhaoqing University, Zhaoqing, China \\ Email: hmh9520@sina.com
}

How to cite this paper: Huang, M.H. (2019) Interfacial Crack Problem of a Class of Spliced Materials. Journal of Applied Mathematics and Physics, 7, 2811-2817. https://doi.org/10.4236/jamp.2019.711193

Received: October 17, 2019

Accepted: November 17, 2019

Published: November 20, 2019

Copyright (c) 2019 by author(s) and Scientific Research Publishing Inc. This work is licensed under the Creative Commons Attribution International License (CC BY 4.0).

http://creativecommons.org/licenses/by/4.0/

\begin{abstract}
The interfacial crack problem of a class of spliced materials is discussed. Using plane elastic complex variable method and integral equation theory, one method of solving the complex stress functions is given.

\section{Keywords}

Interfacial Crack, Complex Variable Method, Boundary Value Problems, Integral Equation Theory
\end{abstract}

\section{Introduction}

Composite materials are widely used in engineering practice. During the long-term use of composite materials, phenomena such as holes and cracks will occur, causing damage to composite materials. Cracking problem of composite materials, especially the problem of interfacial crack has been the topic of considerable research during the past decades, for instance [1] [2] [3] [4].

However, to the best of our knowledge, various types of interfacial crack problems have not been fully studied. The aim of this paper is to study the stress state of one splicing problem of a strip and a half-plane of isotropic materials with interfacial cracks. By employing plane elastic complex variable method and theory of boundary value problems for analytic functions, using proper decomposition of the functions and integral transformation, the problem is reduced to a singular integral equation of normal type. The existence and uniqueness of solution were proved. Further, using the integral equation theory, the closed form solution of stress function was given.

For definiteness and simplicity, we will only discuss the first fundamental problem and the case of a single crack. 


\section{Formulation of Problems and Stress Function}

Let the elastic body occupy the lower half-plane $Z^{-}$. The elastic body spliced by two dissimilar isotropic materials, one is a strip $S^{+}(h \leq y \leq 0,|x|<+\infty, h>0)$, the other is a semi-infinity plane $S^{-}\left(S^{-}=Z^{-}-S^{+}\right)$, corresponding elastic constants are $\kappa^{+}, \mu^{+}$and $\kappa^{-}, \mu^{-}$, respectively. There is a crack $r=\overline{a b}$ locate on the splicing line $L(y=-h i,|x|<+\infty)$. We will discuss the first fundamental problem, that is finding the elastic equilibrium, under known the external stress $X_{n}^{ \pm}(t)+i Y_{n}^{ \pm}(t) \in H \quad$ (one class of Hölder continuous functions) on both sides of $r$ and $X_{0}^{ \pm}(t)+i Y_{0}^{ \pm}(t) \in \hat{H} \quad$ (one class of Hölder continuous functions on infinity line) on $X$-axis. Besides, the stresses and the angle of rotation at infinity is also given. Obviously, the principal vectors of the external stresses on $r^{ \pm}$and on $X$ is $X^{ \pm}+i Y^{ \pm}=\int_{r}\left[X_{n}^{ \pm}(t)+i Y_{n}^{ \pm}(t)\right] \mathrm{d} t$ and $X_{0}+i Y_{0}=\int_{X}\left[X_{0}(x)+i Y_{0}(x)\right] \mathrm{d} x$ respectively.

Our problem is how to determine the complex potentials describing the stress state of elastic body.

Let $\varphi(z)$ and $\Omega(z)$ is the complex stress functions for the elastic body. Because the elastic body is in balance state, without loss of generality, we can assume the principle vectors of stress on both $X$ and $r$ is zero, and no stresses or rotation at infinity. Thus, the stress functions $\varphi(z)$ and $\Omega(z)$ is sectionally holomorphic in $S^{+}+S^{-}$with $\varphi(\infty)=\Omega(\infty)=0$.

According to the conditions known above and the theory of elastic complex variable [5], our problem boils down to the following boundary value problem with functions $\varphi(z)$ and $\Omega(z)$ :

$$
\begin{gathered}
\varphi(x)+\overline{\Omega(x)}=f_{1}(x), x \in X . \\
\varphi^{ \pm}(t)-2 h \overline{i \varphi^{\prime \pm}(t)}+\overline{\Omega^{ \pm}(t)}=f_{2}^{ \pm}(t)+C, t \in r . \\
\varphi^{+}(t)-2 h \overline{i \varphi^{\prime+}(t)}+\overline{\Omega^{+}(t)}=\varphi^{-}(t)-2 h i \overline{\varphi^{\prime}(t)}+\overline{\Omega^{-}(t)}, t \in l=L-r . \\
\alpha^{+} \varphi^{+}(t)-\beta^{+}\left[-2 h \overline{\varphi^{\prime+}(t)}+\overline{\Omega^{+}(t)}\right] \\
=\alpha^{-} \varphi^{-}(t)-\beta^{-}\left[-2 h i \overline{\varphi^{\prime-}(t)}+\overline{\Omega^{-}(t)}\right], t \in l=L-r .
\end{gathered}
$$

where

$$
\begin{aligned}
& \alpha^{ \pm}=\kappa^{ \pm} / \mu^{ \pm}, \beta^{ \pm}=1 / \mu^{ \pm}, \\
& f_{1}(x)=i \int_{-\infty}^{x}\left[X_{0}(x)+i Y_{0}(x)\right] \mathrm{d} x, f_{2}^{ \pm}(t)=i \int_{a}^{t}\left[X_{n}^{ \pm}(t)+i Y_{n}^{ \pm}(t)\right] \mathrm{d} t,
\end{aligned}
$$

$C$ is undetermined constant.

\section{Solving Boundary Value Problem}

For solving the above boundary value problem (2.1)-(2.4), we introduce a new function:

$$
\omega(t)=\varphi^{+}(t)-\varphi^{-}(t), t \in L .
$$

Hence $\omega(t), \omega^{\prime}(t) \in H$ and $\omega(a)=\omega(b)=0$. 
From Equations (2.2) and (2.3), we have

$$
\Omega^{+}(t)-\Omega^{-}(t)=\left\{\begin{array}{l}
\overline{F(t)}-\overline{\omega(t)}-2 h i \omega^{\prime}(t), t \in r \\
\overline{\omega(t)}-2 h i \omega^{\prime}(t), t \in l .
\end{array}\right.
$$

Hence $F(t)=f^{+}(t)-f^{-}(t)$.

Defination:

$$
\begin{gathered}
\varphi_{1}(z)=\frac{1}{2 \pi i} \int_{L} \frac{\omega(t)}{t-z} \mathrm{~d} t, z \notin L . \\
\Omega_{1}(z)=-\frac{1}{2 \pi i} \int_{L} \frac{\overline{\omega(t)}+2 h i \omega^{\prime}(t)}{t-z} \mathrm{~d} t+\frac{1}{2 \pi i} \int_{r} \frac{\overline{F(t)}}{t-z} \mathrm{~d} t, z \notin L .
\end{gathered}
$$

Obviously, $\varphi_{1}(z)$ and $\Omega_{1}(z)$ is sectionally holomorpic functions defined in a full plane, with line of jump $L$ and $\varphi(\infty)=\Omega_{1}(\infty)=0$.

Using Plemelj's integral formula, from Equations (3.2) and (3.3), we get

$$
\varphi_{1}^{+}(t)-\varphi^{+}(t)=\varphi_{1}^{-}(t)-\varphi^{-}(t), \Omega_{1}^{+}(t)-\Omega^{+}(t)=\Omega_{1}^{-}(t)-\Omega^{-}(t), t \in L .
$$

According to Equation (3.5), we can define two holomorpic functions of the entire lower half plane ( $\operatorname{Imz}<0$ ), which continuous to $X$-axis as following:

$$
\varphi_{2}(z)=\varphi_{1}(z)-\varphi(z), z \in Z^{-} ; \Omega_{2}(z)=\Omega_{1}(z)-\Omega(z), z \in Z^{-} .
$$

Substituting Equation (3.6) into Equation (2.1), we have

$$
\varphi_{1}(x)-\varphi_{2}(x)+\overline{\Omega_{1}(x)-\Omega_{2}(x)}=f_{1}(x), x \in X .
$$

Multiplying both sides of Equation (3.7) and its conjugating equation by $\frac{1}{2 \pi i} \cdot \frac{\mathrm{d} x}{x-z}(\operatorname{Im} z<0)$ respectively, and then integrating along the $X$-axis. Considering the previously given conditions and Cauchy's integral theorem, we get

$$
\varphi_{2}(z)=\overline{\Omega_{1}(\bar{z})}+\frac{1}{2 \pi i} \int_{X} \frac{f_{1}(x)}{x-z} \mathrm{~d} x, \Omega_{2}(z)=\overline{\varphi_{1}(\bar{z})}+\frac{1}{2 \pi i} \int_{X} \frac{\overline{f_{1}(x)}}{x-z} \mathrm{~d} x .
$$

From Equations (3.6), (3.8), (3.3), (3.4), we have

$$
\begin{aligned}
\varphi(z)= & \frac{1}{2 \pi i} \int_{L} \frac{\omega(t)}{t-z} \mathrm{~d} t-\frac{1}{2 \pi i} \int_{L} \frac{\omega(t)-2 h i \overline{\omega^{\prime}(t)}}{\bar{t}-z} \mathrm{~d} \bar{t} \\
& +\frac{1}{2 \pi i} \int_{r} \frac{F(t)}{\overline{t-z}} \mathrm{~d} \bar{t}-\frac{1}{2 \pi i} \int_{X} \frac{f_{1}(x)}{x-z} \mathrm{~d} x, z \notin L . \\
\Omega(z)= & \frac{1}{2 \pi i} \int_{L} \frac{\overline{\omega(t)}}{\bar{t}-z} \mathrm{~d} \bar{t}-\frac{1}{2 \pi i} \int_{L} \frac{\overline{\omega(t)}+2 h i \omega^{\prime}(t)}{t-z} \mathrm{~d} t \\
& +\frac{1}{2 \pi i} \int_{r} \frac{\overline{F(t)}}{t-z} \mathrm{~d} t-\frac{1}{2 \pi i} \int_{X} \frac{\overline{f_{1}(x)}}{x-z} \mathrm{~d} x, z \notin L .
\end{aligned}
$$

Substituting Equations (3.9) and (3.10) into Equation (2.2), either for positive or negative boundary value, we obtain the same equation as following:

$$
\begin{gathered}
\frac{1}{\pi i} \int_{L} \frac{\omega(\tau)}{\tau-t} \mathrm{~d} \tau-\frac{1}{2 \pi i} \int_{L} \omega(\tau) \mathrm{d}\left[\ln (\tau-\bar{t})(\bar{\tau}-t)-\frac{(t-\bar{t})(\tau-\bar{\tau})}{(\tau-\bar{t})^{2}}\right] \\
-\frac{1}{2 \pi i} \int_{L} \overline{\omega(\tau)} \mathrm{d}\left[\frac{\bar{\tau}-\tau}{\bar{\tau}-t}+\frac{t-\bar{t}}{\tau-\bar{t}}\right]=g_{1}(t)+C, t \in r .
\end{gathered}
$$


where

$$
\begin{aligned}
g_{1}(t)= & \frac{1}{2}\left[f^{+}(t)+f^{-}(t)\right]-\frac{h}{\pi} \int_{X}\left[\frac{f_{1}(x)}{(x-t)(x-\bar{t})}+\frac{\overline{f_{1}(x)}}{(x-\bar{t})^{2}}\right] \mathrm{d} x \\
& -\frac{1}{2 \pi i} \int_{r}\left[\frac{F(\tau)}{\bar{\tau}-t}-\frac{\overline{F(\tau)}}{(x-\bar{t})^{2}}\right] \mathrm{d} \tau
\end{aligned}
$$

Substituting Equations (3.9), (3.10) into Equations (2.3), the equation satisfies automatically. Substituting them into Equation (2.4), we get

$$
\begin{aligned}
& A \omega(t)+\frac{B}{\pi i} \int_{L} \frac{\omega(\tau)}{\tau-t} \mathrm{~d} \tau+\frac{1}{\pi i} \int_{L} \omega(\tau) \mathrm{d}\left[\ln \frac{(\tau-\bar{t})^{D}}{(\bar{\tau}-t)^{E}}-\frac{D(t-\bar{t})(\tau-\bar{\tau})}{(\tau-\bar{t})^{2}}\right] \\
& +\frac{1}{\pi i} \int_{L} \overline{\omega(\tau)} \mathrm{d}\left[\frac{E(\bar{\tau}-\tau)}{\bar{\tau}-t}+\frac{D(t-\bar{t})}{\tau-\bar{t}}\right]=g_{2}(t), t \in l .
\end{aligned}
$$

where we have set

$$
\begin{aligned}
A=\alpha^{+}+\alpha^{-}+ & \beta^{+}+\beta^{-}, B=\alpha^{+}-\alpha^{-}-\beta^{+}+\beta^{-}, D=\beta^{+}-\beta^{-}, E=\alpha^{+}-\alpha^{-}, \\
g_{2}(t)= & -\frac{1}{\pi i} \int_{r}\left[\frac{D(\bar{t}-t) F(\tau)}{(\tau-\bar{t})^{2}} \mathrm{~d} \tau+\left(\frac{E \overline{F(\tau)}}{\bar{\tau}-t}+\frac{D \overline{F(\tau)}}{\overline{\tau-t}}\right) \mathrm{d} \bar{\tau}\right] \\
& -\frac{1}{\pi i} \int_{X}\left(\frac{E f_{1}(x)}{x-t}-\frac{D f_{1}(x)}{x-\bar{t}}-\frac{2 h i D \overline{f_{1}(x)}}{(x-\bar{t})^{2}}\right) \mathrm{d} x .
\end{aligned}
$$

Equations (3.11) and (3.12) constitute a singular integral equation of normal type along $L$.

The following is to prove the existence and uniqueness of the solutions to Equations (3.11) and (3.12). The existence of the equation solution can be explained by the solution of the equation. In order to prove the equation has a unique solution in class $h_{2}$, that is, $\omega(a)$ and $\omega(b)$ are finite. We first show that its corresponding homogeneous equation has only the trivial solution in $h_{2}$. In fact, the latter corresponds to the case where there are no stress on $r$ and $X$, no stresses or rotation at infinity and $C=0$. Assume $\omega(t)$ is a solution of this homogeneous equation. By the uniqueness theorem for elastic problems [5], Since $\varphi(\infty)=\Omega(\infty)=0$, we should have $\varphi(z)=\Omega(z)=0$. Therefore $\omega(t)=\varphi^{+}(t)-\varphi^{-}(t)=0$ for any $t \in L$.

It is easily verified that the index of the singular integral equation Equations (3.11) and (3.12) in class $h_{2}$ is -1 . According to the Noether theorem of integral equations [6], it's adjoint equation has two linearly independent solutions $\sigma_{1}(t), \sigma_{2}(t)(t \in L)$ in class $h_{0}$ (the solutions are permitted to have integrable singularities at the nodes), and it is (uniquely) solvable in this class if and only if [6]

$$
\operatorname{Re} \int_{r}\left[g_{1}(t)+C\right] \sigma_{j}(t) \mathrm{d} t+\operatorname{Re} \int_{l} g_{2}(t) \sigma_{j}(t) \mathrm{d} t=0, j=1,2 .
$$

Once the constant $C$ is determined by equation Equation (3.13), the unique solution $\omega(t) \in h_{2}$ can be deduced by Equations (3.11) and (3.12). Thus, the 
stress functions $\varphi(z)$ and $\Omega(z)$ is obtained, which satisfy boundary value conditions (2.1)-(2.4).

\section{Discussion of Integral Equations}

In practice, it is rather difficult to determine $C$ from Equation (3.13). In fact, we usually only need to know stress distribution of the elastic body. Therefore, it is enough to know only $\varphi^{\prime}(z), \Omega^{\prime}(z)$, that is, to find $W(t)=\omega^{\prime}(t), t \in L$. For this purpose, differentiating Equations (3.11) and (3.12) respectively, we obtain one singular integral equation as following:

$$
\begin{gathered}
\frac{1}{\pi i} \int_{L} \frac{W(\tau)}{\tau-t} \mathrm{~d} \tau+\frac{1}{2 \pi i} \int_{L} W(\tau) k_{1 r}(\tau, t) \mathrm{d} \tau \\
+\frac{1}{2 \pi i} \int_{L} \overline{W(\tau)} k_{2 r}(\tau, t) \mathrm{d} \bar{\tau}=g_{1}^{\prime}(t), t \in r . \\
A W(t)+\frac{B}{\pi i} \int_{L} \frac{W(\tau)}{\tau-t} \mathrm{~d} \tau-\frac{1}{\pi i} \int_{L} W(\tau) k_{1 l}(\tau, t) \mathrm{d} \tau \\
+\frac{1}{2 \pi i} \int_{L} \overline{W(\tau)} k_{2 l}(\tau, t) \mathrm{d} \bar{\tau}=g_{2}^{\prime}(t), t \in l .
\end{gathered}
$$

where $W(t)=\omega^{\prime}(t)$

$$
\begin{aligned}
& k_{1 r}(\tau, t)=\frac{\partial}{\partial t}\left[\ln (\tau-\bar{t})(\bar{\tau}-t)-\frac{(t-\bar{t})(\tau-\bar{\tau})}{(\tau-\bar{t})^{2}}\right], \\
& k_{2 r}(\tau, t)=\frac{\partial}{\partial t}\left[\frac{\bar{\tau}-\tau}{\bar{\tau}-t}+\frac{t-\bar{t}}{\tau-\bar{t}}\right] \\
& k_{1 l}(\tau, t)=\frac{\partial}{\partial t}\left[\ln \frac{(\bar{\tau}-t)^{D}}{(\bar{\tau}-t)^{E}}-\frac{D(t-\bar{t})(\tau-\bar{\tau})}{(\tau-\bar{t})^{2}}\right], \\
& k_{2 l}(\tau, t)=\frac{\partial}{\partial t}\left[\frac{E(\bar{\tau}-\tau)}{\bar{\tau}-t}+\frac{D(t-\bar{t})}{\tau-\bar{t}}\right]
\end{aligned}
$$

Equations (4.1) and (4.2) constitute a singular integral equation on $L$ without constant $C$.

We will give the solution of Equations (4.1) and (4.2) below. It is notice when $\tau, t \in L$, we have $\tau=x-h i, t=x_{0}-h i$ and $\mathrm{d} \tau=\mathrm{d} x, \mathrm{~d} \bar{\tau}=\mathrm{d} x$. Therefore, (4.1) and (4.2) can be changed as

$$
\begin{aligned}
& \alpha(x) W_{*}\left(x_{0}\right)+\frac{\beta(x)}{\pi i} \int_{-\infty}^{+\infty} \frac{W_{*}(x)}{x-x_{0}} \mathrm{~d} x+\int_{-\infty}^{+\infty} W_{*}(x) k_{1 x}\left(x, x_{0}\right) \mathrm{d} x \\
& +\int_{-\infty}^{+\infty} \overline{W_{*}(x)} k_{2 x}\left(x, x_{0}\right) \mathrm{d} x=g\left(x_{0}\right) .
\end{aligned}
$$

where $W_{*}(x)=W(x-h i)$,

$$
\begin{aligned}
& \alpha(x)=\left\{\begin{array}{l}
0, x-h i \in r \\
A, x-h i \in l
\end{array}\right. \\
& \beta(x)=\left\{\begin{array}{l}
1, x-h i \in r \\
B, x-h i \in l
\end{array}\right. \\
& g(x)=\left\{\begin{array}{l}
g_{1}^{\prime}(x-h i), x-h i \in r \\
g_{2}^{\prime}(x-h i), x-h i \in l
\end{array}\right.
\end{aligned}
$$




$$
\begin{aligned}
& k_{1 x}\left(x, x_{0}\right)=\left\{\begin{array}{l}
\frac{1}{2 \pi i} k_{1 r}\left(x-h i, x_{0}-h i\right), x-h i \in r \\
-\frac{1}{\pi i} k_{1 l}\left(x-h i, x_{0}-h i\right), x-h i \in l
\end{array}\right. \\
& k_{2 x}\left(x, x_{0}\right)=\left\{\begin{array}{l}
\frac{1}{2 \pi i} k_{2 r}\left(x-h i, x_{0}-h i\right), x-h i \in r \\
-\frac{1}{\pi i} k_{2 l}\left(x-h i, x_{0}-h i\right), x-h i \in l
\end{array}\right.
\end{aligned}
$$

By the general theory of singular integral equations [6], from Equation (4.3), we get

$$
W_{*}\left(x_{0}\right)+\int_{-\infty}^{+\infty} W_{*}(x) k_{1 x}^{*}\left(x, x_{0}\right) \mathrm{d} x+\int_{-\infty}^{+\infty} \overline{W_{*}(x)} k_{2 x}^{*}\left(x, x_{0}\right) \mathrm{d} x=g_{*}\left(x_{0}\right) .
$$

where

$$
\begin{gathered}
g_{*}(x)=\frac{1}{\alpha^{2}(x)-\beta^{2}(x)}\left[\alpha(x) g(x)-\frac{\beta(x)}{\pi i} \int_{-\infty}^{+\infty} \frac{g(x)}{x-x_{0}} \mathrm{~d} x\right], \\
k_{j x}^{*}\left(x, x_{0}\right) \\
=\frac{1}{\left[\alpha^{2}(x)-\beta^{2}(x)\right] \pi i}\left[\alpha(x) k_{j x}\left(x, x_{0}\right)-\frac{\beta(x)}{\pi i} \int_{-\infty}^{+\infty} \frac{k_{j x}(x, y)}{y-x_{0}} \mathrm{~d} y\right], j=1,2 .
\end{gathered}
$$

Equation (4.4) is a Fredholm's integral equation with continuous and conjugate kernel. According to the theorem of integral equation [6], Equation (4.4) is equivalent to the following system of equations

$$
U\left(x_{0}\right)+\int_{-\infty}^{+\infty} K\left(x, x_{0}\right) U(x) \mathrm{d} x=G\left(x_{0}\right) .
$$

where

$$
U(x)=\left(\frac{W_{*}(x)}{W_{*}(x)}\right), K\left(x, x_{0}\right)=\left(\begin{array}{ll}
\frac{k_{1 x}^{*}\left(x, x_{0}\right)}{k_{2 x}^{*}\left(x, x_{0}\right)} & \frac{k_{2 x}^{*}\left(x, x_{0}\right)}{k_{1 x}^{*}\left(x, x_{0}\right)}
\end{array}\right), G(x)=\left(\frac{g_{*}(x)}{g_{*}(x)}\right) .
$$

From the Equations (4.5), we get

$$
U\left(x_{0}\right)=G\left(x_{0}\right)+\int_{-\infty}^{+\infty} \Gamma\left(x, x_{0} ; 1\right) G(x) \mathrm{d} x .
$$

Hence, $\Gamma\left(x, x_{0} ; 1\right)$ is the solution kernel matrix of kernel matrix $K\left(x, x_{0}\right)$.

Replace $W_{*}(x)$ one by one, the solution of stress functions are obtained in closed form. Therefore, the stress distribution in elastic body is obtained.

\section{Conclusions}

The problems discussed in this paper, as well as similar problems, are often encountered in engineering practice. The solution of the closed form of the problem is obtained by using the classical analysis method; it is helpful for further analysis and precise numerical solution. Compared with the direct use of numerical methods, it has certain advantages.

In addition, the stress intensity factor of crack tip can be further calculated by using the results obtained. The stress intensity factor on the crack tip is of the most important for study the crack propagation and fatigue life. 


\section{Conflicts of Interest}

The author declares no conflicts of interest regarding the publication of this paper.

\section{References}

[1] Monfared, M.M., Ayatollahi, M. and Bagheri, R. (2016) In-Plane Stress Analysis of Dissimilar Materials with Multiple Interface Cracks. Applied Mathematical Modelling, 40, 8464-8474. https://doi.org/10.1016/j.apm.2016.05.006

[2] Abe, M., Ikeda, T., Koganemaru, M., et al. (2017) Stress Intensity Factor Analysis of a Three-Dimensional Interfacial Corner between Anisotropic Piezoelectric Multi-Materials under Several Boundary Conditions on the Corner Surfaces. Engineering Fracture Mechanics, 171, 1-21.

https://doi.org/10.1016/j.engfracmech.2016.12.009

[3] Doblare, M., Espiga, F., Gracia, L., et al. (1990) Study of Crack Propagation in Orthotropic Materials by Using the Boudary Element Method. Engineering Fracture Mechanics, 37, 953-967. https://doi.org/10.1016/0013-7944(90)90020-H

[4] Lu, J.K. (1993) A General Method for Solving Plane Crack Problems. Journal of Integral Equations and Applications, 5, 85-101.

https://doi.org/10.1216/jiea/1181075729

[5] Muskhelishvili, N.I. (1953) Some Basic Problem in the Mathematical Theory of Elasticity. Noordhoff, Groningen.

[6] Muskhelishvili, N.I. (1962) Singular Integral Equations. Noordhoff, Groningen. 